OPEN ACCESS

Edited by:

Xiaoqiang Tang,

Sichuan University, China

Reviewed by:

Ding Ai,

Tianjin Medical University, China

Hou-Zao Chen,

Chinese Academy of Medical

Sciences and Peking Union Medical

College, China

*Correspondence:

Yaqian Huang

yaqianhuang@126.com

Hongfang Jin

jinhongfang@bjmu.edu.cn;

jinhongfang51@126.com

${ }^{\dagger}$ These authors have contributed equally to this work

Specialty section:

This article was submitted to

Cellular Biochemistry,

a section of the journal

Frontiers in Cell and Developmental

Biology

Received: 04 November 2020

Accepted: 26 January 2021

Published: 18 February 2021

Citation:

Peng $H$, Wang $X, D u J$, Cui $Q$, Huang $Y$ and Jin $H$ (2021) Metabolic

Reprogramming of Vascular

Endothelial Cells: Basic Research

and Clinical Applications.

Front. Cell Dev. Biol. 9:626047.

doi: $10.3389 /$ fcell.2021.626047

\section{Metabolic Reprogramming of Vascular Endothelial Cells: Basic Research and Clinical Applications}

\author{
Hanlin Peng ${ }^{1 \dagger}$, Xiuli Wang ${ }^{1 \dagger}$, Junbao Du ${ }^{1,2}$, Qinghua Cuil ${ }^{2,3}$, Yaqian Huang ${ }^{1 *}$ and \\ Hongfang Jin ${ }^{1 *}$
}

' Department of Pediatrics, Peking University First Hospital, Beijing, China, ${ }^{2}$ Key Laboratory of Molecular Cardiovascular Sciences, Ministry of Education, Beijing, China, ${ }^{3}$ Department of Biomedical Informatics, Centre for Non-coding RNA Medicine, School of Basic Medical Sciences, Peking University, Beijing, China

Vascular endothelial cells (VECs) build a barrier separating the blood from the vascular wall. The vascular endothelium is the largest endocrine organ, and is well-known for its crucial role in the regulation of vascular function. The initial response to endothelial cell injury can lead to the activation of VECs. However, excessive activation leads to metabolic pathway disruption, VEC dysfunction, and angiogenesis. The pathways related to VEC metabolic reprogramming recently have been considered as key modulators of VEC function in processes such as angiogenesis, inflammation, and barrier maintenance. In this review, we focus on the changes of VEC metabolism under physiological and pathophysiological conditions.

\footnotetext{
Keywords: energy metabolism, metabolic reprogramming, endothelial cell dysfunction, glycolysis, mitochondrial oxidation
}

Vascular endothelium has a variety of physiological functions, for instance, serving as a barrier between blood and tissues and acting as an endocrine organ in the body (Krüger-Genge et al., 2019). Indeed, vascular endothelial cells (VECs) can synthesize and release various endothelial-derived vasoactive factors to regulate blood flow, coagulation, and fibrinolytic balance. VEC activation is defined as stimulus-induced quantitative changes in the expression of specific genes (Pober, 1988), and excessive activation causes endothelial cell dysfunction or unnecessary angiogenesis. VEC activation and dysfunction are characteristics of atherosclerosis, diabetes, obesity, and aging (Theodorou and Boon, 2018). And in the development of atherosclerosis, VEC activation and dysfunction in vulnerable areas of arterial vessels are among the earliest changes that can be detected (Davignon and Ganz, 2004). Moreover, tumor neovascularization is characterized by a highly disordered vascular network and the generation of new blood vessels. Despite in-depth studies of cellular metabolism, the metabolic properties of VECs have only recently been a focus of research (Eelen et al., 2018). It is found that changes in VEC metabolism lead to endothelial cell dysfunction and are closely related with the pathogenesis of many diseases such as atherosclerosis, diabetic angiopathy, pulmonary hypertension, etc. (Pober et al., 2009). Based on these findings, VEC metabolism is becoming a new therapeutic target for various diseases. Nevertheless, the relationship between VEC metabolism and the pathogenesis of vascular dysfunction and remodeling remains incompletely understood. Overall, in this review, we briefly summarize the current understanding of the metabolic changes in dysfunctional VECs, provide a detailed overview of the metabolic pathways activated under normal and pathological conditions, in order to identify potential therapeutic targets on the VEC metabolic reprogramming. 


\section{VEC METABOLISM UNDER NORMAL PHYSIOLOGICAL CONDITION}

Theoretically, since VECs are exposed to oxygen from the bloodstream, they are expected to produce adenosine triphosphate (ATP) through oxidative phosphorylation (OXPHOS) for energy-yielding. Nevertheless, due to the relatively low mitochondrial content of VECs, energy production is still mainly dependent of glycolysis which yields more than $85 \%$ of ATP under normal physiological condition (De Bock et al., 2013b). Conversely, glucose-derived pyruvate enters the mitochondria to participate in the metabolism of glucose via the tricarboxylic acid cycle (TCA), resulting in the production of ATP, which is less than 1\% (De Bock et al., 2013b). Although the glycolysis-derived ATP by per mole glucose is low, ATP generated from glycolysis in a short time period is more quickly than that from OXPHOS in the presence of an unrestricted amount of glucose (Eelen et al., 2015), because VECs have a higher glycolytic rate than many other healthy cell types (De Bock et al., 2013b). VEC aerobic glycolysis also has the following advantages over other processes: (1) a reduction of reactive oxygen species (ROS) produced by OXPHOS; (2) the ability to maximize oxygen transfer to cells surrounding the blood vessel; (3) the ability to adapt to hypoxic environments, and (4) the production of lactate, which can promote angiogenesis (Eelen et al., 2015).

Another advantage of glycolysis is the possible shunt of glucose to side branches, including the hexosamine biosynthesis pathway (HBP), pentose phosphate pathway (PPP), and polyol pathway (PP) for biomacromolecule synthesis. Of all the glucose utilized by VECs, only 1-3\% enters the PPP under physiological condition (De Bock et al., 2013a). The PPP leads to the generation of nicotinamide adenine dinucleotide phosphate (NADPH) and ribose-5-phosphate (R5P). NADPH can convert oxidized glutathione (GSSG) to glutathione (GSH) and maintain the internal redox balance. And R5P can be used to synthesize nucleotides (De Bock et al., 2013a). On the other hand, although the role of the HBP in VECs in vivo is not clear, $\mathrm{N}$-acetylglucosamine produced by this pathway is an essential substance for $\mathrm{N}$-glycosylation and $\mathrm{O}$-glycosylation and may be the key to the glycosylation of angiogenic proteins (Luo et al., 2008). Finally, when the amount of glucose exceeds the capacity of glycolysis, glucose enters the PP, where glucose is catalyzed to sorbitol by aldose reductase (AR), and later is converted to fructose. Since AR requires NADPH to provide reducing power, PP activation consumes NADPH, thus leading to the accumulation of ROS (De Bock et al., 2013a).

In addition to glucose, fatty acids (FAs) are another energy source for VECs. FAs can be converted into acetyl-CoA, and the latter can be used to generate reducing power and produce ATP in the mitochondria. However, the VEC mitochondria serve as a signaling switching station such as mitochondrial calcium signaling, ROS generation from electron transport chain and NO production catalyzed by eNOS, rather than power factories (Davidson, 2010; Groschner et al., 2012). Therefore, fatty acid oxidation (FAO) occurring in the mitochondria may not contribute substantially to total ATP production in VECs.
Though mitochondrial is not that important for providing energy, the disturbed mitochondrial dynamics contributes to VEC dysfunction and the development of vascular diseases (Tang et al., 2014). Kalucka et al. found that FAO in the silence ECs is important for maintaining redox homeostasis and EC functions (Kalucka et al., 2018), while FAO in proliferative ECs is also indispensable for de novo dNTP synthesis (Schoors et al., 2015).

To date, the metabolism of amino acid in VECs is not well studied except for arginine and glutamine metabolism. Nitric oxide (NO), a crucial modulator of VEC function, is generated from arginine by endothelial nitric oxide synthase (eNOS) (Tousoulis et al., 2012). Interestingly, the conversion of glutamine into glucosamine inhibits the activity of the oxidative PPP (oxPPP), thereby leading to the reduction of NADPH (an important cofactor for eNOS) and inhibiting the production of endothelial NO (Wu et al., 2001). Moreover, glutamine-derived glutamate can be the substrate to be used in the other nonessential amino acids production. It also acts as a supplementary carbon source in the TCA cycle for ATP production after further metabolized into $\alpha$-ketoglutarate (Kim B. et al., 2017).

In summary, the main energy sources of VECs is derived from glucose glycolysis, whereas FAO and glutamine oxidation are generally thought to supplement the TCA cycle via OXPHOS (Figure 1). When the rate of glycolysis decreases, the energy provided by the oxidative metabolism of glucose, FAs, and amino acids might alternatively increase for supporting the VEC activity (Krutzfeldt et al., 1990).

\section{METABOLIC REPROGRAMMING IN DYSFUNCTIONAL VECS}

Many factors can activate VECs, such as lipopolysaccharide (LPS), interleukin 1 (IL-1) and tumor necrosis factor (TNF- $\alpha$ ) (Magnuson et al., 1989). After activation, VEC metabolism is disordered, represented by the increased glycolysis, and increased expression level and activity of fatty acid synthase (FAS) (Pan et al., 2009; De Bock et al., 2013b; Feng et al., 2017; Singh et al., 2017; Li et al., 2019b). These enhance the proliferation, migration and inflammation of VECs, leading to VEC dysfunction and vascular diseases. In the next paragraphs, we will review in detail the metabolic changes and VEC dysfunction.

\section{Metabolic Reprogramming and VEC Migration}

VECs migrate to an anoxic microenvironment in the tumor angiogenesis development. The energy supporting the above process is mainly derived from VEC anaerobic metabolism. Therefore, the migrated VECs are highly dependent of glycolysis compared with silence VECs. In VECs, glycolysis normally occurs in the perinuclear cytoplasm; however, once these cells begin to migrate, glycolysis also takes place in lamellipodia and filopodia to promote the rapid production of ATP needed for migration (De Bock et al., 2013b). Glucose-6-phosphate dehydrogenase (G6PD) is the rate-limiting enzyme of PPP, and the overexpression of G6PD will stimulate VEC migration due to the increase in NO and NADPH production (Pan et al., 


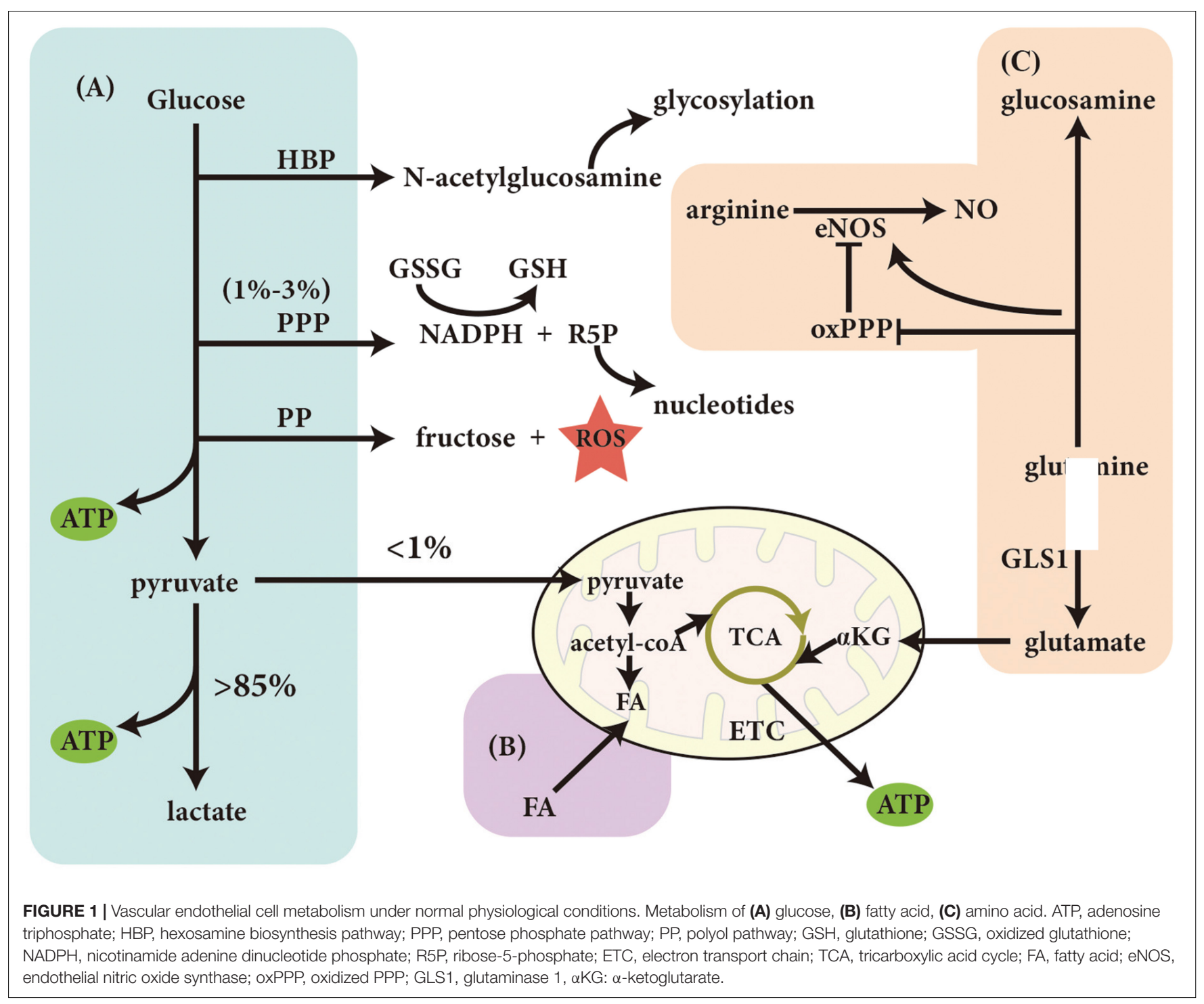

2009). Conversely, an increased glucosamine concentration leads to protein glycosylation and inhibits the migration of VECs (Li et al., 2019b).

\section{Metabolic Reprogramming and VEC Inflammation}

The metabolic changes of inflammatory VECs are mainly characterized by increased glycolysis. The study showed that mechanical low shear stress activated hypoxia-inducible factor $1 \alpha(\mathrm{HIF}-1 \alpha)$ in cultured VECs via activating the nuclear factor- $\kappa \mathrm{B}(\mathrm{NF}-\kappa \mathrm{B})$ pathway and promoting the expression of deubiquitinating enzyme cezanne. HIF-1 $\alpha$ promotes the production of inflammatory factors in VECs by increasing the expression of the glycolysis-related regulators hexokinase 2 (HK2), enolase 2 (ENO2), glucose transporter 1 (GLUT1), fructose-2,6-biphosphatase 3 (PFKFB3) and extracellular acidification rate (ECAR, a direct marker of glycolysis) (Feng et al., 2017).

\section{Metabolic Reprogramming and VEC Proliferation}

Studies have found that multiple steps in FA metabolism, such as FA synthesis, FA transport and FAO, are all involved in the proliferation of VECs. Firstly, the expression and activity of FAS are increased in hypoxic human pulmonary artery endothelial cells (HPAECs), while the inhibition of FAS leads to the reduced HPAEC proliferation (Singh et al., 2017). Secondly, VEC proliferation is associated with the expression of fatty acid transporters (FATPs) and fatty acid binding proteins (FABPs). For instance, the silencing of FABP4 inhibited the VEC proliferation in vitro (Elmasri et al., 2012). Also, the downregulation of carnitine palmitoyl transferase 1A (CPT1A) expression, a ratelimiting enzyme in the metabolism of FA, or CPT1A knockout was also found to inhibit the proliferation of VECs (Schoors et al., 2015). The above results indicate that fatty acid metabolism plays a significant role in controlling VEC proliferation. However, the detailed mechanisms need further studies. 


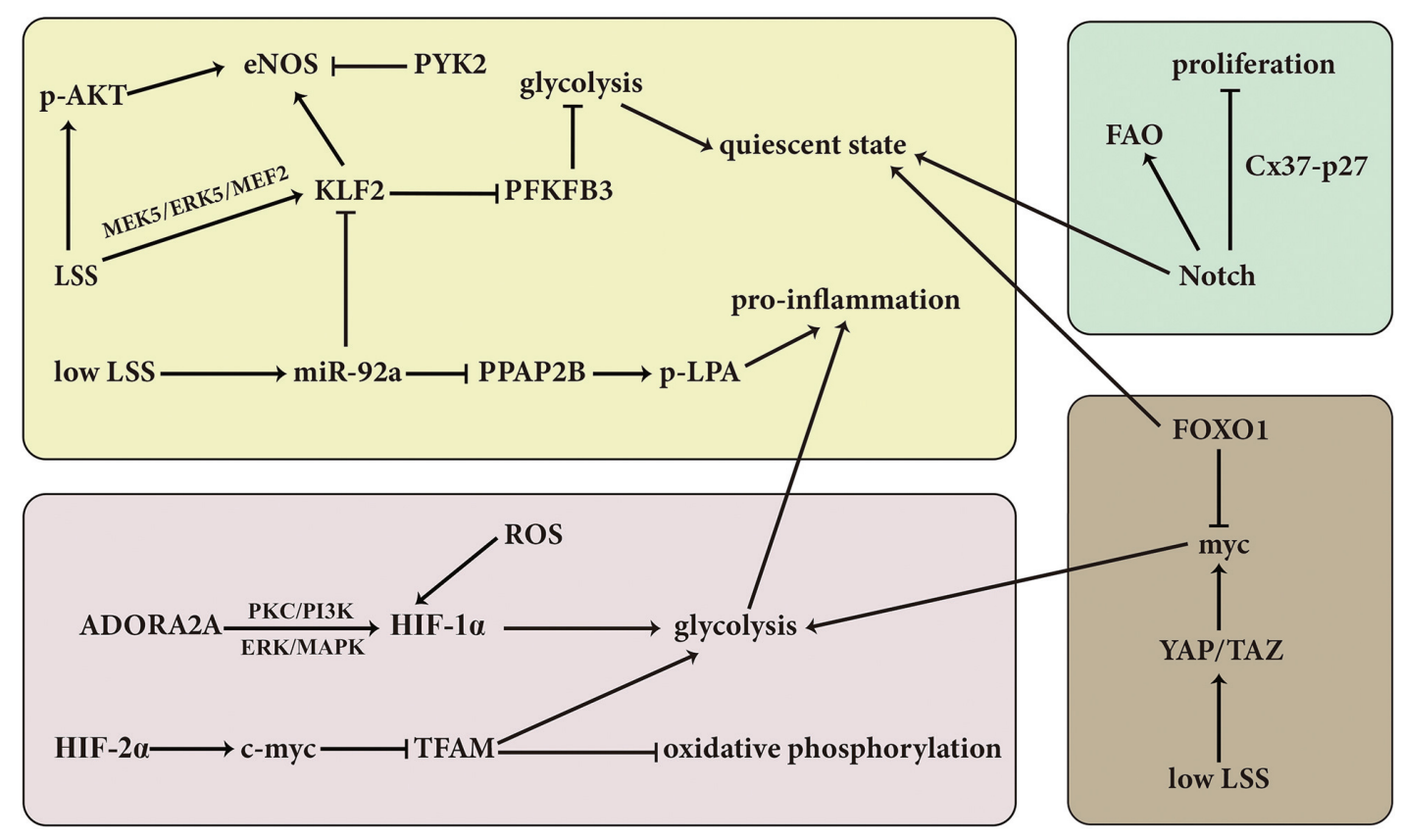

FIGURE 2 | Overview of the molecular mechanisms and signaling pathways involved in the regulation of VEC. LSS, laminar shear stress; KLF2, Kruppel-like factor 2; PFKFB3, fructose-2,6-biphosphatase 3; PPAP2B, phosphatidic acid phosphatase type 2B; LPA, lysophosphatidic acid; AKT, protein kinase B; eNOS, endothelial nitric oxide synthase; PYK2, proline-rich tyrosine kinase 2; ROS, reactive oxygen species; HIF-1 $\alpha$, hypoxia inducible factor-1 $\alpha$; PKC, protein kinase C; ADORA2A, adenosine A2a receptor; FOXO1, forkhead box O1; FAO, fatty acid oxidation; YAP, Yes-associated protein; TAZ, transcriptional coactivator with PDZ-binding motif; p, phosphorylation.

The proliferation of VECs also depends on glutamine metabolism. Indeed, genetic deletion or pharmacological inhibition of glutaminase1(GLS1) inhibits the proliferation of VECs (Kim B. et al., 2017). Asparagine synthetase (ASNS) is an enzyme which is responsible for the generation of asparagine from nitrogen (glutamine-derived) and aspartic acid. Huang et al. found that the silencing of ASNS inhibited the proliferation of VECs (Huang et al., 2017).

\section{MOLECULAR MECHANISMS AND SIGNALING PATHWAYS INVOLVED IN THE REGULATION OF VEC METABOLISM}

Various signaling pathways are involved in the regulation of VEC metabolism to respond quickly to changes under environmental conditions (Figure 2; Li et al., 2019c).

Laminar shear stress (LSS) is mainly transduced via the activation of a mechanical signal transduction pathway triggering a certain response of VECs to the external environment; at the same time, LSS can also affect VEC metabolism. It is reported that the expression of transcriptional factor Kruppel like factor 2 (KLF2) is induced in the LSS-treated VECs via the activation of MEK5/ERK5/MEF2 signaling pathway (Parmar et al., 2006). Subsequently, a great number of genes controlling endothelial energy metabolism, thrombosis/hemostasis, inflammation, vascular tone, and vessel development/remodeling are regulated by the LSS-induced KLF2 pathway (SenBanerjee et al., 2004; Parmar et al., 2006). For example, PFKFB3 is a key glycolytic enzyme. It was found that LSS-upregulated KLF2 reduced glucose uptake and glycolysis by inhibiting the activity of the PFKFB3 promoter, then decreased the flux of glycolysis and maintained VECs at a resting state (Dekker et al., 2002; Doddaballapur et al., 2015). On the other hand, under normal condition, phosphatidic acid phosphatase type $2 \mathrm{~B}$ (PPAP2B) dephosphorylates lysophosphatidic acid (LPA) to prevent it from binding to its receptor LPAR1 and then inhibits VEC inflammation. While, low LSS leads to the increased miR-92a and decreased KLF2, which inhibits the expression of PPAP2B in VECs, and then induces the VEC inflammation (Wu et al., 2011, 2015). Another key point in the response to LSS is the activity of eNOS. ECs possess special membrane organelle cilia, which is responsible for sensing the LSS and thus controlling the production of NO (Nauli et al., 2008). However, the underlying mechanisms by which LSS regulates the activity of eNOS are quite sophisticated. For example, LSS-induced phosphorylation of AKT and increased expression of KLF2 can activate and promote the expression of eNOS, respectively, but upregulation of proline-rich tyrosine kinase 2(PYK2) leads to opposite result (Zhou et al., 2014).

Like KLF2, SIRT3 also regulates the metabolic shift between mitochondrial oxidation and glycolysis, targeting on the PFKFB3. It was found that SIRT3 KO-EC exhibited a reduction of glycolysis and an elevation in mitochondrial oxidation and ROS 
formation, accompanying with the downregulated expression of PFKFB3 and upregulated acetylation of PFKFB3. The abovementioned EC metabolic shift was regarded to contribute to an impaired angiogenesis, reduced coronary flow reserve and diastolic dysfunction in SIRT3 KO mice (He et al., 2017).

Some studies have suggested that hypoxia signaling also participates in the regulation of VEC metabolism. For example, the upregulation of pyruvate dehydrogenase kinase 1(PDK1) induced by HIF-1 in the disturbed flow-activated ECs results in the phosphorylation and inactivation of pyruvate dehydrogenase $(\mathrm{PDH})$, then blocks the conversion from pyruvate to acetylCoA catalyzed by $\mathrm{PDH}$, and finally suppresses the TCA cycle. At the same time, the increased glucose transporter and glycolytic enzyme genes such as GLUT1, lactate dehydrogenase A (LDHA) and HK-2 due to the activation of HIF-1 pathway are also involved in the disturbed flow-activated EC metabolic reprogramming and inflammation (Prabhakar and Semenza, 2012; Wu et al., 2017). Moreover, in hypoxic pulmonary artery ECs, HIF-2 $\alpha$ inhibits mitochondrial transcription factor A (TFAM) by downregulating the expression of c-myc, and thus leads to the suppression of mitochondrial gene expression and the decrease in oxidative phosphorylation (Zarrabi et al., 2017). Regarding the mechanism responsible for the activation of HIF signaling, NAD(P)H Oxidase-4 (NOX4)-derived ROS is found to be required for the disturbed flow-activated HIF-1 pathway and subsequently increased glycolysis and decreased mitochondrial respiration ( $\mathrm{Wu}$ et al., 2017). Besides, protein kinase C (PKC) and PI3K signaling are also involved in the hypoxia-induced HIF$1 \alpha$ activation and reinforced glycolysis in hypoxic VECs (Paik et al., 2017). Except for the above signaling pathways, adenosine A2a receptor (ADORA2A) in hypoxic VECs can enhance HIF-1 $\alpha$ protein synthesis via ERK/MAPK and PI3K/Akt pathways and therefore promote glycolytic enzyme expression, glycolytic flux, EC proliferation, and angiogenesis (Liu et al., 2017).

The activation of Notch signaling pathway is necessary for maintaining the quiescent state of VECs. Kalucka et al. found that the treatment with Notch pathway stimulator Dll4 in the proliferating ECs induced a significant quiescence of ECs represented by the fact that the treated ECs became less proliferative and more in G0 phase. Simultaneously, the transcriptome results showed that the expression of genes related with glycolysis, TCA cycle, nucleotide synthesis, and purine/pyrimidine synthesis were decreased while the expression of genes controlling FAO was increased (Kalucka et al., 2018). Furthermore, it was found that Notch signaling led to the increased FAO flux, which was utilized for redox homeostasis through the generation of NADPH in quiescent VECs. The abovementioned effect of Notch signaling was mediated by the upregulation of CPTA1 (Kalucka et al., 2018). Moreover, Notch signaling is activated in the fluid shear stress-induced EC quiescence model, in which Cx37-p27 serve as the downstream of Notch signaling to promote the EC cell cycle arrest (Fang et al., 2017).

Similarly, forkhead box O1 (FOXO1) also is an important switch bridging the EC growth status and metabolic activities (Wilhelm et al., 2016). FOXO1 is found to decrease glycolysis, reduce mitochondrial respiration, inhibit EC proliferation and sprouting angiogenesis, and thereby maintain the EC in a quiescence status. EC-specific deletion of FOXO1, however, results in an uncontrolled EC proliferation, vessel hyperplasia and enlargement. Furthermore, it is found that the constitutive activation of FOXO1 reduces the myc expression, promotes myc degradation, and increases the expression of the negative regulators of myc MXI1. Considering that myc is a strong driver of glycolysis, mitochondrial metabolism, and cell growth, the antagonization of myc might mediate the pro-quiescence effect of FOXO1. FOXO1/myc acts a novel metabolic gatekeeper for switching the EC proliferation to quiescence (Wilhelm et al., 2016). On the contrary, endothelial Yes-associated protein/transcriptional coactivator with PDZ-binding motif complex (YAP/TAZ) promotes the formation and maturation of brain vessel in mice by upregulating myc-drive glycolysis, mitochondrial oxidative phosphorylation and EC proliferation (Kim J. et al., 2017). Low LSS activates YAP/TAZ by enhancing the activity of c-Jun N-terminal kinase (Wang et al., 2016), and the activation of integrin $\alpha 5 \beta 1$ and $c-A b l$ induced by low LSS can upregulate the nuclear translocation of YAP by phosphorylating YAP at Y357 (Li et al., 2019a). Additionally, the RAF-MEK-ERK pathway activates glutaminolysis in VECs to support the metabolic requirements of highly proliferative VECs (Guo et al., 2016).

\section{VEC METABOLISM UNDER PATHOLOGICAL CONDITION}

Under normal physiological conditions, the main energy source of VECs comes from glycolysis. However, the increased activity of glycolytic-related enzymes after VEC activation can trigger the pro-inflammatory pathway, contributing to the progress of vascular injury diseases like atherosclerosis. Conversely, when the activity of glycolysis-related enzymes is reduced, resulting in stagnated glycolysis, intermediate products accumulate and can be metabolized via other pathways. The consequent generation of a great number of oxidative substances, the generation of advanced glycation end product (AGE), and eNOS uncoupling can lead to diabetic angiopathy. Furthermore, VECs frequently show a high glycolytic phenotype while retaining functional mitochondria during tumor neovascularization. Finally, metabolic reprogramming of VECs is also involved in the pathogenesis of hypertension and pulmonary arterial hypertension (Figure 3 ). In the following paragraphs, we shall review in further detail the abnormal VEC metabolism and its significance under pathophysiological conditions.

\section{VEC Metabolism in Atherosclerosis}

One of the most crucial factors for the initiation of VEC activation is disturbed blood flow dynamics (Davignon and Ganz, 2004). The disturbed blood flow mainly leads to the metabolic changes of VEC glycolysis. As mentioned in the above text, the expression of transcription factor KLF2 is upregulated by high LSS, resulting in decreased glycolysis and mitochondrial respiration by inhibiting the promoter of 


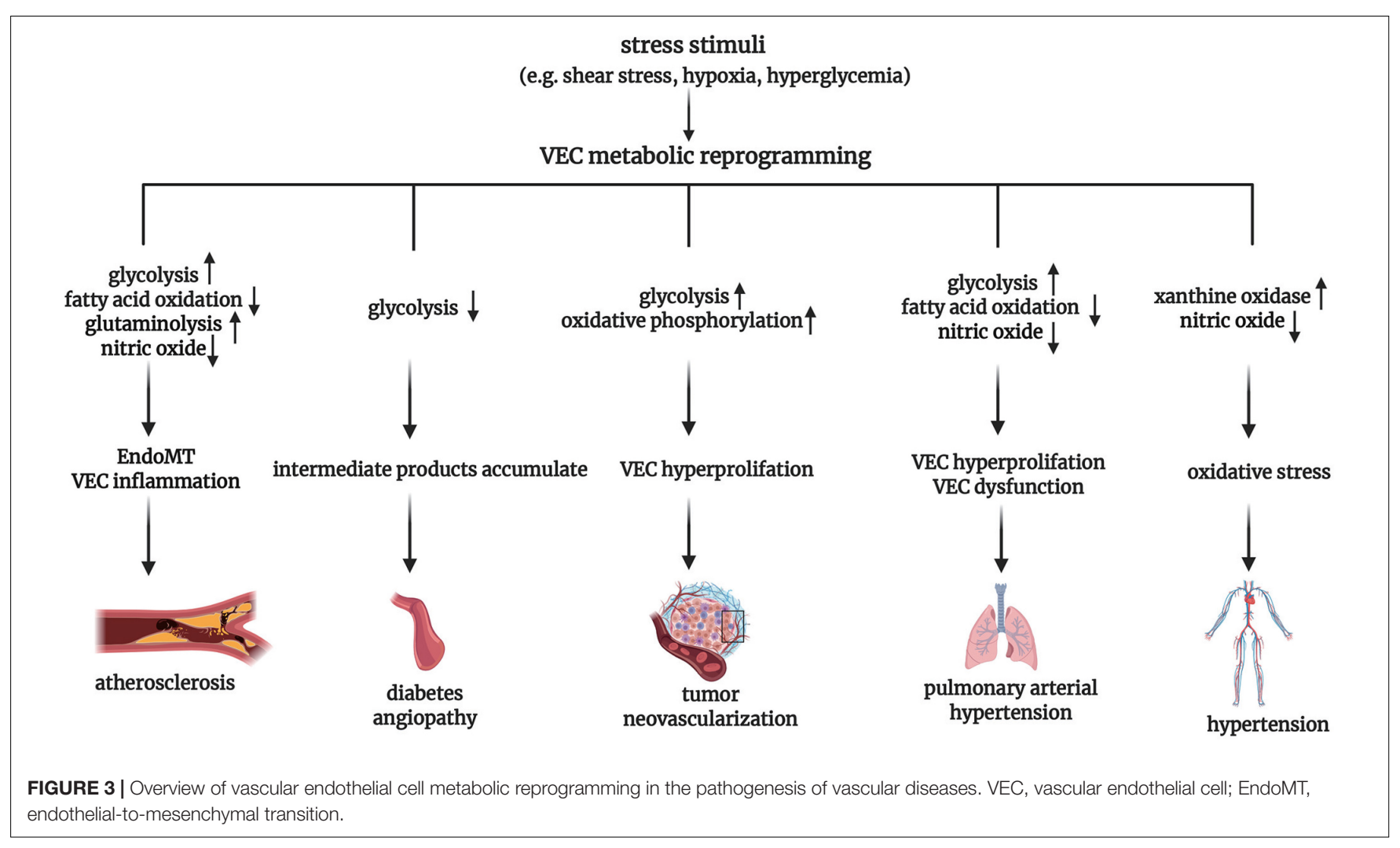

PFKFB3 (Doddaballapur et al., 2015). As a result, KLF2 is thought to be a key transcriptional switch point regulating the quiescent and activated states of VECs. On the other hand, VECs in the atherosclerotic region of the vascular system are disrupted by low LSS. Under low LSS, the activation of NF- $\kappa \mathrm{B}$ induces elevated HIF- $1 \alpha$ mRNA and increased the expression of cezanne, a ubiquitin-editing enzyme preventing the HIF$1 \alpha$ protein degradation, thus stabilizing HIF-1 $\alpha$. And then, HIF- $1 \alpha$ promotes the proliferation and inflammation of VECs by activating glycolytic genes $\mathrm{HK} 2, \mathrm{ENO} 2$, and PFKFB3 to upregulate glycolysis, resulting in the initiation of atherosclerosis (Feng et al., 2017).

Endothelial-to-mesenchymal transition (EndoMT) is an important pathological basis of atherosclerotic lesions. These VEC-derived mesenchymal cells can cause the instability of plaque by enhancing the expression and activity of collagenmatrix metalloproteinase (Evrard et al., 2016). Xiong et al. demonstrated a metabolic mechanism responsible for EndoMT targeting on the FAO-controlled acetyl-CoA. It was found that the induction of EndoMT was associated with a reduction in FAO demonstrated by a decrease in acetyl-CoA levels and a fall in the expression of CPT1A, the enzyme that played rate-limiting and obligate roles in FAO. The downregulation of CPT1A expression in ECs promoted the EndoMT, while the increase in acetyl-CoA levels by supplementing acetate inhibited the SMAD2 activation and EndoMT program in cytokine-stimulated ECs. Those abovementioned results suggest the causal relationship between the reduction of FAO in the
ECs and EndoMT, and provide a novel metabolic therapeutic choice for EndoMT-related diseases such as atherosclerosis (Xiong et al., 2018).

It has been proved that the pro-inflammatory YAP/TAZ signaling promotes the glutaminolysis of VECs, suggesting that glutamine has an atherogenic effect (Bertero et al., 2016). Glutamine deficiency can lead to the increased endoplasmic reticulum stress, impaired TCA, inhibition of mTOR signaling and subsequently reduced protein synthesis (Huang et al., 2017). Moreover, glutamate can be converted to neurotransmitter gamma aminobutyric acid (GABA), an anti-inflammatory mediator, in HAECs and HUVECs, and therefore glutamine deficiency may reduce the GABA level and its anti-inflammatory effect (Sen et al., 2016). In atherosclerosis, VECs also show disruption in metabolic pathways related to NO production. In the endothelium, NO-mediated vasodilation is required for vascular homeostasis and the inhibition of important events that promote atherosclerosis, including platelet aggregation, and smooth muscle cells migration (Kawashima, 2004). Consistently, one of the early features of atherosclerosis is eNOS uncoupling, resulting in an imbalance between anti-atherosclerotic NO and pro-atherosclerotic peroxides (Pircher et al., 2016).

\section{VEC Metabolism in Diabetic Angiopathy}

As a key metabolic characteristic of diabetes, hyperglycemia is found to be closely correlated with the alterations of 
VEC metabolism, VEC dysfunction and consequent diabetic angiopathy. A significantly increased production of ROS and reactive nitrogen species (RNS) is an important change of diabetic VEC metabolism (Goveia et al., 2014). Hyperglycemia leads to an increased ROS level in VEC mainly by three mechanisms: (1) an obviously increased expression and activity of $\mathrm{NAD}(\mathrm{P}) \mathrm{H}$ oxidase protein subunits (p22phox, p67phox, and p47phox), which was observed in the veins and arteries of diabetic patients (Guzik et al., 2002). The NADPH-derived ROS production is associated with the downregulation of 8 oxoguanine glycosylase and subsequent activation of PKC in high glucose-treated HUVECs (Xie et al., 2020); (2) xanthine oxidase (XO) inhibitor allopurinol can prevent hyperglycemiainduced generation of ROS, which indicates that $\mathrm{XO}$ may be the major contributor of ROS (Eleftheriadis et al., 2018); (3) uncoupled eNOS can induce the increase of oxidative stress in diabetic mice (Sasaki et al., 2008). Besides, hyperglycemia results in an increase in CAMP, and then through the CAMPdependent PKA, G6PD is phosphorylated and its activity is inhibited. As a result, the entry of glucose into PPP is reduced, leading to the subsequent decreased NADPH and increased ROS (Zhang et al., 2000). Moreover, when exposed to high glucose, the expression of fission proteins fission-1 (Fis1) and dynamin-related protein-1 (Drp1) in the VEC mitochondrial is enhanced and leads to the increase in the mitochondrial fission and an impaired autophagy of mitochondrial. And then the altered mitochondrial dynamics results in an increase of ROS generation, an inhibition of eNOS activation, and the loss of NO bioavailability (Shenouda et al., 2011).

Excess ROS production in diabetic VECs can activate polyadenosine diphosphate ribose polymerase 1 (PARP-1), which mediates the ribosylation of poly-ADP, thereby inactivating the key glycolytic enzyme glyceraldehyde triphosphate dehydrogenase (GAPDH), whose activity plays a vital role in the maintenance of glycolytic flux (Du et al., 2003). As a result, the inhibited GAPDH leads to the stalled glycolysis. Excess glucose that cannot be metabolized due to the stalled glycolysis enters the $\mathrm{PP}$ and is converted to sorbitol at the cost of NADPH by AR, thereby leading to an increase in ROS. Sorbitol is then switched to fructose and highly active 3deoxyglucose (3DG), promoting the formation of AGEs (Goveia et al., 2014). And the accumulated glycolysis intermediates then turn to three abnormal metabolic pathways as follows. (1) Glycolytic-derived fructose 6-phosphate can produce fructose-6-phosphate (F6P) via 6-phosphate fructosyl amide transferase; and the accumulated F6P is then mainly metabolized through the HBP, which produces the important precursor of glycosylation, uracil- $N$-acetylglucosamine diphosphate (UDP-GlcNAc) (Brownlee, 2001). Although glycosylation is an important process for the physiological function of VECs, hyperglycemic-induced protein glycosylation may inhibit angiogenesis (Luo et al., 2008). (2) The intermediates of glycolysis glyceraldehyde 3-phosphate (G3P) and dihydroxyacetone phosphate (DHAP) are transferred to the methylglyoxal pathway, which further boosts the production of AGEs (Goveia et al., 2014). Moreover, G3P and DHAP contribute to the diacylglycerol de novo synthesis, and subsequent $\mathrm{PKC}$ activation causes vascular abnormalities (Das Evcimen and King, 2007). (3) In theory, the accumulated glucose-6-phosphate (G6P) can enter the glucuronate cycle, but there is a lack of comprehensive research on the glucuronate cycle in diabetic VECs (Eelen et al., 2018).

\section{VEC Metabolism in Tumor Neovascularization}

The vessels in tumor are highly abnormal, and mainly characterized by VEC hyperproliferation. The switch of VECs from the quiescent state to the proliferative and migratory state during tumor neovascularization is closely related with the VEC metabolic reprogramming. VECs in tumors show a greater dependence on glycolysis to produce ATP than healthy VECs, mainly manifested as increased expression levels of associated genes, such as the glucose transporter GLUT1 and the glycolytic activator $P F K F B 3$, and thus exhibit a high glycolytic phenotype. The upregulation of PFKFB3 in the tumor depends on hypoxia, pro-inflammatory cytokines, or hormone signaling in the microenvironment (Zecchin et al., 2017). In addition, PPP and the serine biosynthetic pathway, which are utilized for the synthesis of nucleotide and biomass, are more highly activated in tumor VECs than in healthy VECs (Rohlenova et al., 2018).

Both tumor and healthy VECs retain functional mitochondria. The active OXPHOS increases the flexibility of using other substrates for energy generation, and provides metabolites for the synthesis of biomass to support cell proliferation. Studies revealed that the death of proliferating tumor VECs caused by mitochondrial respiratory inhibition is more than that of quiescent VECs, which indicated that functional OXPHOS may be quite important for the proliferating tumor VECs (Rohlenova et al., 2018). The role of mitochondria in tumor endothelial cells deserves further study.

\section{VEC Metabolism in Pulmonary Arterial Hypertension}

The high proliferation and dysfunction of VECs are main characteristics of pulmonary arterial hypertension (PAH) (Yu and Chan, 2017). The excessive proliferation of VECs in PAH depends on an increased glycolysis flux and a decreased oxygen consumption related to the upregulation of HIF-1 $\alpha$ (Tuder et al., 2012). Moreover, bone morphogenetic protein receptor type 2 (BMPR2) is an important human $\mathrm{PAH}$ pathogenic gene (Majka et al., 2011). The expression of a BMPR2 mutant protein in human lung VECs contributes to the progress of PAH mainly by subsequent metabolic changes in VECs: (1) upregulated expression of glycolysis-related enzymes; (2) depressed carnitine metabolism and fatty acid oxidation; and (3) decreased TCA cycle intermediates (Fessel et al., 2012). In addition, the increase in isocitrate dehydrogenase (IDH)1 and IDH-2 activity observed in BPMR2-mutated VECs is identical with the increased serum IDH activity of PAH patients (Fessel et al., 2012). 
Another characteristic of PAH is the decrease of NO content in VECs, which may be related to the following two aspects: (1) the decrease in antioxidant manganese superoxide dismutase (MnSOD) in mitochondria since MnSOD increases the biological activity of NO by scavenging the superoxide anion (Fijalkowska et al., 2010); and (2) the high expression level of arginase II, which competes with eNOS for the substrate arginine, thus leading to the subsequent decreased production of NO (Xu et al., 2004).

\section{VEC Metabolism in Hypertension}

In addition to the above EC metabolic changes, the abnormal purine catabolism in VECs is involved in the EC dysfunction in the development of hypertension. Xanthine oxidoreductase (XOR) catalyzes the oxidation of hypoxanthine to xanthine, and then xanthine to uric acid (UA). XOR exists in two different forms: xanthine dehydrogenase (XD) and XO (Maruhashi et al., 2018). The differences between $\mathrm{XD}$ and $\mathrm{XO}$ include the following aspects: (1) XD is the main enzyme found in normal tissues, but its activity is low, and XO dominates in tissues with injury and ischemia (Schmitz and Brand, 2016). (2) They use different electron acceptors. $\mathrm{NAD}^{+}$is preferentially used in the XD catalyzed-reaction, in which a stable reaction product $\mathrm{NADH}$ is generated; while molecular oxygen is preferentially used in the $\mathrm{XO}$ catalyzed-reaction, resulting the generation of superoxide anion and hydrogen peroxide (Maruhashi et al., 2018).

Compared with Wistar-Kyoto rat, $\mathrm{XO}$ activity was increased in VECs of spontaneously hypertensive rat (SHR), which was related with an elevation of arteriolar tone (Vila et al., 2019). Besides ROS generated from XO catalyzed-reaction, the concomitant product UA is regarded to be closely related with EC dysfunction and hypertension. Correspondingly, UA-lowering drugs have a significant anti-hypertensive effect via protecting EC in the basic experiment and clinical trials (Otani et al., 2018; Yang et al., 2018). The proposed mechanisms responsible for UA-related EC dysfunction involved the followings: (1) the impairment NO production and bioavailability. UA inhibited phosphorylation of eNOS and production of NO via PI3K/Akt pathway (Choi et al., 2014). Also, UA suppressed NO release and reacted directly with $\mathrm{NO}$ in an irreversible manner leading to NO depletion (Kang et al., 2005; Gersch et al., 2008). (2) the pro-inflammatory effect. UA treatment in VECs upregulated the mRNA expression, protein and release of inflammatory mediator C-reactive protein via p38 and ERK pathway (Kang et al., 2005). (3) the impaired mitochondrial function. UA-treated human aortic ECs exhibited the reduction mitochondrial mass and ATP production, the decrease in aconitase- 2 activity and expression of enoyl CoA hydratase1 (Sánchez-Lozada et al., 2012). (4) the induction of EC phenotype transition. UA-induced EndoMT in HUVECs via ROS generation and glycocalyx shedding (Ko et al., 2019). (5) The inhibition of EC proliferation and migration (Gersch et al., 2008). However, it is worth noticing that UA is also an antioxidant. Its main properties include scavenging hydroxyl free radicals, superoxide anions, peroxynitrite and preventing lipid peroxidation (Amaro et al., 2019).

\section{KEY INSIGHTS AND THERAPEUTIC PERSPECTIVE}

In summary, VECs mostly remain quiescent, but can be activated by various physiological and pathological stimuli. In general, VECs show significant metabolic changes during activation, resulting in further VEC dysfunction and development of cardiovascular disease, diabetic angiopathy, and tumor angiogenesis. For example, the activation and dysfunction of VECs in vulnerable areas of arterial blood vessels can be easily detected in the development of atherosclerosis (Davignon and Ganz, 2004), accompanied by unique metabolic reprogramming of VECs (Goveia et al., 2014). Moreover, vascular dysfunction caused by VEC metabolic changes initiates the development of diabetic angiopathy and contributes to the pathogenesis of vascular complications (Shi and Vanhoutte, 2017). In addition to vascular growth factors, VEC metabolism also plays a significant role in tumor neovascularization (Rohlenova et al., 2018). The abovementioned studies indicate that VEC metabolism might be a possible therapeutic target of vasculature related diseases.

At present, one of the important strategies targeting VEC metabolism is the development of anti-angiogenic drugs (LopesCoelho et al., 2020). PFKFB3 is an important enzyme in glycolysis, and participates in the synthesis of ATP and biological macromolecules. Initial study has demonstrated that the inhibition of the gene PFKFB3 may reduce the formation of vessels under physiological condition (De Bock et al., 2013b). Notably, anti-glycolysis therapy with the PFKFB3 blocker 3(3-pyridinyl)-1-(4-pyridinyl)-2-propen-1-one(3PO) can reduce glycolysis and pathological angiogenesis (Schoors et al., 2014), and a phase 1 clinical trial of the 3PO derivative PFK158 for the treatment of solid malignant tumor has been carried out (Li et al., 2019b). Therapy targeting PFKFB3 is safe because it temporarily inhibits only a branch of glycolysis and does not harm other healthy tissues that depend on glycolysis for their energy production (Schoors et al., 2014). Besides, Schoonjans et al. have demonstrated that PDK inhibitor dichloroacetate (DCA) and GLS1 inhibitor Bis-2-(5-phenylacetamido-1,3,4thiadiazol-2-yl) ethyl sulfide (BPTES) affect tumor angiogenesis by lowering glycolysis and glutamate production, respectively, in HUVECs (Schoonjans et al., 2020). The silence of CPT1 in VECs can lead to the proliferation of VECs. As a result, it suggests that the pharmacological blockade of CPT1, such as etomoxir, may be used for the treatment of pathological angiogenesis by lowering FAO (Schoors et al., 2015). Further strategies to recover abnormal metabolism, decrease ROS production, and enhance the clearance of ROS, seem to be able to help counteracting diabetic vascular disease initiated by VEC dysfunction. However, clinical antioxidants have not been successfully applied to treat diabetic complication, to some extent (Ceriello et al., 2016). Therefore, new therapeutic targets are a continued focus of research related to vascular complications in diabetes. Nonetheless, VEC-based metabolic targeted therapies for atherosclerosis had not been developed to date.

As our understanding of VEC metabolism improves, new treatment options may continue to emerge. The detailed characterization of VEC metabolism under normal 
and pathophysiological conditions, direct metabolic flux measurements, and more comprehensive metabolomics analyses may provide future treatment targets for a wide range of pathologies related to abnormal vascular development.

\section{AUTHOR CONTRIBUTIONS}

HP sorted out, reviewed, and analyzed the literatures, drew the diagrams, and wrote the manuscript. XW sorted out, reviewed, and analyzed the literatures and revised the manuscript. $\mathrm{HJ}$

\section{REFERENCES}

Amaro, S., Jiménez-Altayó, F., and Chamorro, Á (2019). Uric acid therapy for vasculoprotection in acute ischemic stroke. Brain. Circ. 5, 55-61. doi: 10.4103/ bc.bc_1_19

Bertero, T., Oldham, W. M., Cottrill, K. A., Pisano, S., Vanderpool, R. R., Yu, Q., et al. (2016). Vascular stiffness mechanoactivates YAP/TAZ-dependent glutaminolysis to drive pulmonary hypertension. J. Clin. Invest. 126, 3313-3335. doi: 10.1172/JCI86387

Brownlee, M. (2001). Biochemistry and molecular cell biology of diabetic complications. Nature 414, 813-820. doi: 10.1038/414813a

Ceriello, A., Testa, R., and Genovese, S. (2016). Clinical implications of oxidative stress and potential role of natural antioxidants in diabetic vascular complications. Nutr. Metab. Cardiovasc. Dis. 26, 285-292. doi: 10.1016/j. numecd.2016.01.006

Choi, Y. J., Yoon, Y., Lee, K. Y., Hien, T. T., Kang, K. W., Kim, K. C., et al. (2014). Uric acid induces endothelial dysfunction by vascular insulin resistance associated with the impairment of nitric oxide synthesis. FASEB. J. 28, 31973204. doi: 10.1096/fj.13-247148

Das Evcimen, N., and King, G. L. (2007). The role of protein kinase C activation and the vascular complications of diabetes. Pharmacol. Res. 55, 498-510. doi: 10.1016/j.phrs.2007.04.016

Davidson, S. M. (2010). Endothelial mitochondria and heart disease. Cardiovasc. Res. 88, 58-66. doi: $10.1093 / \mathrm{cvr} / \mathrm{cvq} 195$

Davignon, J., and Ganz, P. (2004). Role of endothelial dysfunction in atherosclerosis. Circulation 109, III27-III32. doi: 10.1161/01.CIR.0000131515. 03336.f8

De Bock, K., Georgiadou, M., and Carmeliet, P. (2013a). Role of endothelial cell metabolism in vessel sprouting. Cell Metab. 18, 634-647. doi: 10.1016/j.cmet. 2013.08.001

De Bock, K., Georgiadou, M., Schoors, S., Kuchnio, A., Wong, B. W., Cantelmo, A. R., et al. (2013b). Role of PFKFB3-driven glycolysis in vessel sprouting. Cell 154, 651-663. doi: 10.1016/j.cell.2013.06.037

Dekker, R. J., van Soest, S., Fontijn, R. D., Salamanca, S., de Groot, P. G., VanBavel, E., et al. (2002). Prolonged fluid shear stress induces a distinct set of endothelial cell genes, most specifically lung Krüppel-like factor (KLF2). Blood 100, 16891698. doi: 10.1182/blood-2002-01-0046

Doddaballapur, A., Michalik, K. M., Manavski, Y., Lucas, T., Houtkooper, R. H., You, X., et al. (2015). Laminar shear stress inhibits endothelial cell metabolism via KLF2-mediated repression of PFKFB3. Arterioscler. Thromb. Vasc. Biol. 35, 137-145. doi: 10.1161/ATVBAHA.114.304277

Du, X., Matsumura, T., Edelstein, D., Rossetti, L., Zsengellér, Z., Szabó, C., et al. (2003). Inhibition of GAPDH activity by poly(ADP-ribose) polymerase activates three major pathways of hyperglycemic damage in endothelial cells. J. Clin. Invest. 112, 1049-1057. doi: 10.1172/jci200318127

Eelen, G., de Zeeuw, P., Simons, M., and Carmeliet, P. (2015). Endothelial cell metabolism in normal and diseased vasculature. Circ. Res. 116, 1231-1244. doi: 10.1161/CIRCRESAHA.116.302855

Eelen, G., de Zeeuw, P., Treps, L., Harjes, U., Wong, B. W., and Carmeliet, P. (2018). Endothelial cell metabolism. Physiol. Rev. 98, 3-58. doi: 10.1152/ physrev.00001.2017

Eleftheriadis, T., Pissas, G., Antoniadi, G., Liakopoulos, V., and Stefanidis, I. (2018). Allopurinol protects human glomerular endothelial cells from high devised the concept. JD, QC, YH, and HJ supervised the writing. All authors revised and approved the final version of the manuscript.

\section{FUNDING}

This research was funded by the National Natural Science Foundation of China (81970424 and 81921001) and Beijing Natural Science Foundation (7171010, 7191012, and 7182168).

glucose-induced reactive oxygen species generation, p53 overexpression and endothelial dysfunction. Int. Urol. Nephrol. 50, 179-186. doi: 10.1007/s11255017-1733-5

Elmasri, H., Ghelfi, E., Yu, C. W., Traphagen, S., Cernadas, M., Cao, H. M., et al. (2012). Endothelial cell-fatty acid binding protein 4 promotes angiogenesis: role of stem cell factor/c-kit pathway. Angiogenesis 15, 457-468. doi: 10.1007/ s10456-012-9274-0

Evrard, S. M., Lecce, L., Michelis, K. C., Nomura-Kitabayashi, A., Pandey, G., Purushothaman, K. R., et al. (2016). Endothelial to mesenchymal transition is common in atherosclerotic lesions and is associated with plaque instability. Nat. Commun. 7:11853. doi: 10.1038/ncomms 11853

Fang, J. S., Coon, B. G., Gillis, N., Chen, Z., Qiu, J., Chittenden, T. W., et al. (2017). Shear-induced Notch-Cx37-p27 axis arrests endothelial cell cycle to enable arterial specification. Nat. Commun. 8:2149. doi: 10.1038/s41467-017-01742-7

Feng, S., Bowden, N., Fragiadaki, M., Souilhol, C., Hsiao, S., Mahmoud, M., et al. (2017). Mechanical activation of hypoxia-inducible factor lalpha drives endothelial dysfunction at atheroprone sites. Arterioscler. Thromb. Vasc. Biol. 37, 2087-2101. doi: 10.1161/ATVBAHA.117.309249

Fessel, J. P., Hamid, R., Wittmann, B. M., Robinson, L. J., Blackwell, T., Tada, Y., et al. (2012). Metabolomic analysis of bone morphogenetic protein receptor type 2 mutations in human pulmonary endothelium reveals widespread metabolic reprogramming. Pulm. Circ. 2, 201-213. doi: 10.4103/2045-8932. 97606

Fijalkowska, I., Xu, W., Comhair, S. A., Janocha, A. J., Mavrakis, L. A., Krishnamachary, B., et al. (2010). Hypoxia inducible-factorlalpha regulates the metabolic shift of pulmonary hypertensive endothelial cells. Am. J. Pathol. 176, 1130-1138. doi: 10.2353/ajpath.2010.090832

Gersch, C., Palii, S. P., Kim, K. M., Angerhofer, A., Johnson, R. J., and Henderson, G. N. (2008). Inactivation of nitric oxide by uric acid. Nucleosides. Nucleotides. Nucleic Acids 27, 967-978. doi: 10.1080/15257770802257952

Goveia, J., Stapor, P., and Carmeliet, P. (2014). Principles of targeting endothelial cell metabolism to treat angiogenesis and endothelial cell dysfunction in disease. EMBO Mol. Med. 6, 1105-1120. doi: 10.15252/emmm.201404156

Groschner, L. N., Waldeck-Weiermair, M., Malli, R., and Graier, W. F. (2012). Endothelial mitochondria-less respiration, more integration. Pflugers Arch. 464, 63-76. doi: 10.1007/s00424-012-1085-z

Guo, Y., Deng, Y., Li, X., Ning, Y., Lin, X., Guo, S., et al. (2016). Glutaminolysis was induced by TGF-betal through PP2Ac regulated Raf-MEK-ERK signaling in endothelial cells. PLoS One 11:e0162658. doi: 10.1371/journal.pone. 0162658

Guzik, T. J., Mussa, S., Gastaldi, D., Sadowski, J., Ratnatunga, C., Pillai, R., et al. (2002). Mechanisms of increased vascular superoxide production in human diabetes mellitus: role of $\mathrm{NAD}(\mathrm{P}) \mathrm{H}$ oxidase and endothelial nitric oxide synthase. Circulation 105, 1656-1662. doi: 10.1161/01.cir.0000012748.58444.08

He, X., Zeng, H., Chen, S. T., Roman, R. J., Aschner, J. L., Didion, S., et al. (2017). Endothelial specific SIRT3 deletion impairs glycolysis and angiogenesis and causes diastolic dysfunction. J. Mol. Cell Cardiol. 112, 104-113. doi: 10.1016/ j.yjmcc.2017.09.007

Huang, H., Vandekeere, S., Kalucka, J., Bierhansl, L., Zecchin, A., Brüning, U., et al. (2017). Role of glutamine and interlinked asparagine metabolism in vessel formation. EMBO J. 36, 2334-2352. doi: 10.15252/embj.201695518

Kalucka, J., Bierhansl, L., Conchinha, N. V., Missiaen, R., Elia, I., Bruning, U., et al. (2018). Quiescent endothelial cells upregulate fatty acid beta-oxidation 
for vasculoprotection via redox homeostasis. Cell Metab. 28, 881.e13-894.e13. doi: 10.1016/j.cmet.2018.07.016

Kang, D. H., Park, S. K., Lee, I. K., and Johnson, R. J. (2005). Uric acid-induced C-reactive protein expression: implication on cell proliferation and nitric oxide production of human vascular cells. J. Am. Soc. Nephrol. 16, 3553-3562. doi: 10.1681/asn.2005050572

Kawashima, S. (2004). Malfunction of vascular control in lifestyle-related diseases: endothelial nitric oxide (NO) synthase/NO system in atherosclerosis. J. Pharmacol. Sci. 96, 411-419. doi: 10.1254/jphs.fmj04006x6

Kim, B., Li, J., Jang, C., and Arany, Z. (2017). Glutamine fuels proliferation but not migration of endothelial cells. EMBO J. 36, 2321-2333. doi: 10.15252/embj. 201796436

Kim, J., Kim, Y. H., Kim, J., Park, D. Y., Bae, H., Lee, D. H., et al. (2017). YAP/TAZ regulates sprouting angiogenesis and vascular barrier maturation. J. Clin. Invest. 127, 3441-3461. doi: 10.1172/jci93825

Ko, J., Kang, H. J., Kim, D. A., Kim, M. J., Ryu, E. S., Lee, S., et al. (2019). Uric acid induced the phenotype transition of vascular endothelial cells via induction of oxidative stress and glycocalyx shedding. FASEB. J. 33, 13334-13345. doi: 10.1096/fj.201901148R

Krüger-Genge, A., Blocki, A., Franke, R. P., and Jung, F. (2019). Vascular endothelial cell biology: an update. Int. J. Mol. Sci. 20:4411. doi: 10.3390/ ijms20184411

Krutzfeldt, A., Spahr, R., Mertens, S., Siegmund, B., and Piper, H. M. (1990). Metabolism of exogenous substrates by coronary endothelial cells in culture. J. Mol. Cell Cardiol. 22, 1393-1404. doi: 10.1016/0022-2828(90)90984-a

Li, B., He, J., Lv, H., Liu, Y., Lv, X., Zhang, C., et al. (2019a). c-Abl regulates YAPY357 phosphorylation to activate endothelial atherogenic responses to disturbed flow. J. Clin. Invest. 129, 1167-1179. doi: 10.1172/jci122440

Li, X., Kumar, A., and Carmeliet, P. (2019b). Metabolic pathways fueling the endothelial cell drive. Annu. Rev. Physiol. 81, 483-503. doi: 10.1146/annurevphysiol-020518-114731

Li, X., Sun, X., and Carmeliet, P. (2019c). Hallmarks of endothelial cell metabolism in health and disease. Cell Metab. 30, 414-433. doi: 10.1016/j.cmet.2019.08.011

Liu, Z., Yan, S., Wang, J., Xu, Y., Wang, Y., Zhang, S., et al. (2017). Endothelial adenosine A2a receptor-mediated glycolysis is essential for pathological retinal angiogenesis. Nat. Commun. 8:584. doi: 10.1038/s41467-017-00551-2

Lopes-Coelho, F., Martins, F., and Serpa, J. (2020). Endothelial cells (ECs) metabolism: a valuable piece to disentangle cancer biology. Adv. Exp. Med. Biol. 1219, 143-159. doi: 10.1007/978-3-030-34025-4_8

Luo, B., Soesanto, Y., and McClain, D. A. (2008). Protein modification by O-linked GlcNAc reduces angiogenesis by inhibiting Akt activity in endothelial cells. Arterioscler. Thromb. Vasc. Biol. 28, 651-657. doi: 10.1161/ATVBAHA.107. 159533

Magnuson, D. K., Maier, R. V., and Pohlman, T. H. (1989). Protein kinase C: a potential pathway of endothelial cell activation by endotoxin, tumor necrosis factor, and interleukin-1. Surgery 106, 216-222. discussion 222-213,

Majka, S., Hagen, M., Blackwell, T., Harral, J., Johnson, J. A., Gendron, R., et al. (2011). Physiologic and molecular consequences of endothelial Bmpr2 mutation. Respir. Res. 12:84. doi: 10.1186/1465-9921-12-84

Maruhashi, T., Hisatome, I., Kihara, Y., and Higashi, Y. (2018). Hyperuricemia and endothelial function: from molecular background to clinical perspectives. Atherosclerosis 278, 226-231. doi: 10.1016/j.atherosclerosis.2018. 10.007

Nauli, S. M., Kawanabe, Y., Kaminski, J. J., Pearce, W. J., Ingber, D. E., and Zhou, J. (2008). Endothelial cilia are fluid shear sensors that regulate calcium signaling and nitric oxide production through polycystin-1. Circulation 117, 1161-1171. doi: 10.1161/circulationaha.107.710111

Otani, N., Toyoda, S., Sakuma, M., Hayashi, K., Ouchi, M., Fujita, T., et al. (2018). Effects of uric acid on vascular endothelial function from bedside to bench. Hypertens Res. 41, 923-931. doi: 10.1038/s41440-018-0095-4

Paik, J. Y., Jung, K. H., Lee, J. H., Park, J. W., and Lee, K. H. (2017). Reactive oxygen species-driven HIFlalpha triggers accelerated glycolysis in endothelial cells exposed to low oxygen tension. Nucl. Med. Biol. 45, 8-14. doi: 10.1016/j. nucmedbio.2016.10.006

Pan, S., World, C. J., Kovacs, C. J., and Berk, B. C. (2009). Glucose 6-phosphate dehydrogenase is regulated through c-Src-mediated tyrosine phosphorylation in endothelial cells. Arterioscler. Thromb. Vasc. Biol. 29, 895-901. doi: 10.1161/ ATVBAHA.109.184812
Parmar, K. M., Larman, H. B., Dai, G., Zhang, Y., Wang, E. T., Moorthy, S. N., et al. (2006). Integration of flow-dependent endothelial phenotypes by Kruppel-like factor 2. J. Clin. Invest. 116, 49-58. doi: 10.1172/jci24787

Pircher, A., Treps, L., Bodrug, N., and Carmeliet, P. (2016). Endothelial cell metabolism: a novel player in atherosclerosis? Basic principles and therapeutic opportunities. Atherosclerosis 253, 247-257. doi: 10.1016/j.atherosclerosis.2016. 08.011

Pober, J. S. (1988). Warner-Lambert/Parke-Davis award lecture. Cytokinemediated activation of vascular endothelium. Physiology and pathology. Am. J. Pathol. 133, 426-433.

Pober, J. S., Min, W., and Bradley, J. R. (2009). Mechanisms of endothelial dysfunction, injury, and death. Annu. Rev. Pathol. 4, 71-95. doi: 10.1146/ annurev.pathol.4.110807.092155

Prabhakar, N. R., and Semenza, G. L. (2012). Adaptive and maladaptive cardiorespiratory responses to continuous and intermittent hypoxia mediated by hypoxia-inducible factors 1 and 2. Physiol. Rev. 92, 967-1003. doi: 10.1152/ physrev.00030.2011

Rohlenova, K., Veys, K., Miranda-Santos, I., De Bock, K., and Carmeliet, P. (2018). Endothelial cell metabolism in health and disease. Trends Cell Biol. 28, 224-236. doi: $10.1016 /$ j.tcb.2017.10.010

Sánchez-Lozada, L. G., Lanaspa, M. A., Cristóbal-García, M., García-Arroyo, F., Soto, V., Cruz-Robles, D., et al. (2012). Uric acid-induced endothelial dysfunction is associated with mitochondrial alterations and decreased intracellular ATP concentrations. Nephron. Exp. Nephrol. 121, e71-e78. doi: 10.1159/000345509

Sasaki, N., Yamashita, T., Takaya, T., Shinohara, M., Shiraki, R., Takeda, M., et al. (2008). Augmentation of vascular remodeling by uncoupled endothelial nitric oxide synthase in a mouse model of diabetes mellitus. Arterioscler. Thromb. Vasc. Biol. 28, 1068-1076. doi: 10.1161/atvbaha.107.160754

Schmitz, B., and Brand, S. M. (2016). Uric acid and essential hypertension: the endothelial connection. J. Hypertens 34, 2138-2139. doi: 10.1097/hjh. 0000000000001109

Schoonjans, C. A., Mathieu, B., Joudiou, N., Zampieri, L. X., Brusa, D., Sonveaux, P., et al. (2020). Targeting endothelial cell metabolism by inhibition of pyruvate dehydrogenase kinase and glutaminase-1. J. Clin. Med. 9:3308. doi: 10.3390/ jcm9103308

Schoors, S., Bruning, U., Missiaen, R., Queiroz, K. C. S., Borgers, G., Elia, I., et al. (2015). Fatty acid carbon is essential for dNTP synthesis in endothelial cells. Nature 526, 192-197. doi: 10.1038/nature14624

Schoors, S., De Bock, K., Cantelmo, A. R., Georgiadou, M., Ghesquiere, B., Cauwenberghs, S., et al. (2014). Partial and transient reduction of glycolysis by PFKFB3 blockade reduces pathological angiogenesis. Cell Metab. 19, 37-48. doi: 10.1016/j.cmet.2013.11.008

Sen, S., Roy, S., Bandyopadhyay, G., Scott, B., Xiao, D., Ramadoss, S., et al. (2016). $\gamma$-aminobutyric acid is synthesized and released by the endothelium: potential implications. Circ. Res. 119, 621-634. doi: 10.1161/CIRCRESAHA.116.308645

SenBanerjee, S., Lin, Z., Atkins, G. B., Greif, D. M., Rao, R. M., Kumar, A., et al. (2004). KLF2 Is a novel transcriptional regulator of endothelial proinflammatory activation. J. Exp. Med. 199, 1305-1315. doi: 10.1084/jem. 20031132

Shenouda, S. M., Widlansky, M. E., Chen, K., Xu, G., Holbrook, M., Tabit, C. E., et al. (2011). Altered mitochondrial dynamics contributes to endothelial dysfunction in diabetes mellitus. Circulation 124, 444-453. doi: 10.1161/ CIRCULATIONAHA.110.014506

Shi, Y., and Vanhoutte, P. M. (2017). Macro- and microvascular endothelial dysfunction in diabetes. J. Diabetes 9, 434-449. doi: 10.1111/1753-0407.12521

Singh, N., Singh, H., Jagavelu, K., Wahajuddin, M., and Hanif, K. (2017). Fatty acid synthase modulates proliferation, metabolic functions and angiogenesis in hypoxic pulmonary artery endothelial cells. Eur. J. Pharmacol. 815, 462-469. doi: 10.1016/j.ejphar.2017.09.042

Tang, X., Luo, Y. X., Chen, H. Z., and Liu, D. P. (2014). Mitochondria, endothelial cell function, and vascular diseases. Front. Physiol. 5:175. doi: 10.3389/fphys. 2014.00175

Theodorou, K., and Boon, R. A. (2018). Endothelial cell metabolism in atherosclerosis. Front. Cell Dev. Biol. 6:82. doi: 10.3389/fcell.2018.00082

Tousoulis, D., Kampoli, A. M., Tentolouris, C., Papageorgiou, N., and Stefanadis, C. (2012). The role of nitric oxide on endothelial function. Curr. Vasc. Pharmacol. 10, 4-18. doi: 10.2174/157016112798829760 
Tuder, R. M., Davis, L. A., and Graham, B. B. (2012). Targeting energetic metabolism: a new frontier in the pathogenesis and treatment of pulmonary hypertension. Am. J. Respir. Crit. Care Med. 185, 260-266. doi: 10.1164/rccm. 201108-1536PP

Vila, E., Solé, M., Masip, N., Puertas-Umbert, L., Amaro, S., Dantas, A. P., et al. (2019). Uric acid treatment after stroke modulates the Krüppel-like factor 2VEGF-A axis to protect brain endothelial cell functions: impact of hypertension. Biochem. Pharmacol. 164, 115-128. doi: 10.1016/j.bcp.2019.04.002

Wang, L., Luo, J. Y., Li, B., Tian, X. Y., Chen, L. J., Huang, Y., et al. (2016). IntegrinYAP/TAZ-JNK cascade mediates atheroprotective effect of unidirectional shear flow. Nature 540, 579-582. doi: 10.1038/nature20602

Wilhelm, K., Happel, K., Eelen, G., Schoors, S., Oellerich, M. F., Lim, R., et al. (2016). FOXO1 couples metabolic activity and growth state in the vascular endothelium. Nature 529, 216-220. doi: 10.1038/nature16498

Wu, C. Q., Huang, R. T., Kuo, C. H., Kumar, S., Kim, C. W., Lin, Y. C., et al. (2015). Mechanosensitive PPAP2B regulates endothelial responses to atherorelevant hemodynamic forces. Circ. Res. 117, E41-E53. doi: 10.1161/Circresaha.117. 306457

Wu, D., Huang, R. T., Hamanaka, R. B., Krause, M., Oh, M. J., Kuo, C. H., et al. (2017). HIF- $1 \alpha$ is required for disturbed flow-induced metabolic reprogramming in human and porcine vascular endothelium. eLife 6:e25217. doi: 10.7554/eLife.25217

Wu, G. Y., Haynes, T. E., Li, H., Yan, W., and Meininger, C. J. (2001). Glutamine metabolism to glucosamine is necessary for glutamine inhibition of endothelial nitric oxide synthesis. Biochem. J. 353, 245-252. doi: 10.1042/0264-6021: 3530245

Wu, W., Xiao, H., Laguna-Fernandez, A., Villarreal, G. Jr., Wang, K. C., Geary, G. G., et al. (2011). Flow-dependent regulation of kruppel-like factor 2 is mediated by microRNA-92a. Circulation 124, 633-641. doi: 10.1161/ circulationaha.110.005108

Xie, X., Chen, Y., Liu, J., Zhang, W., Zhang, X., Zha, L., et al. (2020). High glucose induced endothelial cell reactive oxygen species via OGG1/PKC/NADPH oxidase pathway. Life Sci. 256:117886. doi: 10.1016/j.lfs.2020.117886

Xiong, J., Kawagishi, H., Yan, Y., Liu, J., Wells, Q. S., Edmunds, L. R., et al. (2018). A metabolic basis for endothelial-to-mesenchymal transition. Mol. Cell 69, 689.e7-698.e7. doi: 10.1016/j.molcel.2018.01.010
Xu, W. L., Kaneko, F. T., Zheng, S., Comhair, S. A. A., Janocha, A. J., Goggans, T., et al. (2004). Increased arginase II and decreased NO synthesis in endothelial cells of patients with pulmonary arterial hypertension. FASEB J. 18, 1746-1748. doi: 10.1096/fj.04-2317fje

Yang, H., Bai, W., Gao, L., Jiang, J., Tang, Y., Niu, Y., et al. (2018). Mangiferin alleviates hypertension induced by hyperuricemia via increasing nitric oxide releases. J. Pharmacol. Sci. 137, 154-161. doi: 10.1016/j.jphs.2018. 05.008

Yu, Q., and Chan, S. Y. (2017). Mitochondrial and metabolic drivers of pulmonary vascular endothelial dysfunction in pulmonary hypertension. Adv. Exp. Med. Biol. 967, 373-383. doi: 10.1007/978-3-319-63245-2_24

Zarrabi, A. J., Kao, D., Nguyen, D. T., Loscalzo, J., and Handy, D. E. (2017). Hypoxia-induced suppression of c-Myc by HIF-2alpha in human pulmonary endothelial cells attenuates TFAM expression. Cell Signal. 38, 230-237. doi: 10.1016/j.cellsig.2017.07.008

Zecchin, A., Kalucka, J., Dubois, C., and Carmeliet, P. (2017). How endothelial cells adapt their metabolism to form vessels in tumors. Front. Immunol. 8:1750. doi: 10.3389/fimmu.2017.01750

Zhang, Z., Apse, K., Pang, J., and Stanton, R. C. (2000). High glucose inhibits glucose-6-phosphate dehydrogenase via cAMP in aortic endothelial cells. J. Biol. Chem. 275, 40042-40047. doi: 10.1074/jbc.M007505200

Zhou, J., Li, Y. S., and Chien, S. (2014). Shear stress-initiated signaling and its regulation of endothelial function. Arterioscler. Thromb. Vasc. Biol. 34, 2191-2198. doi: 10.1161/Atvbaha.114.303422

Conflict of Interest: The authors declare that the research was conducted in the absence of any commercial or financial relationships that could be construed as a potential conflict of interest.

Copyright (c) 2021 Peng, Wang, Du, Cui, Huang and Jin. This is an open-access article distributed under the terms of the Creative Commons Attribution License (CC BY). The use, distribution or reproduction in other forums is permitted, provided the original author(s) and the copyright owner(s) are credited and that the original publication in this journal is cited, in accordance with accepted academic practice. No use, distribution or reproduction is permitted which does not comply with these terms. 